\title{
SEPARATION OF TWO CONTRIBUTIONS TO THE HIGH ENERGY EMISSION OF CYGNUS X-1: POLARIZATION MEASUREMENTS WITH INTEGRAL SPI ${ }^{1}$
}

\author{
E. Jourdain ${ }^{1}$, J. P. Roques ${ }^{1}$, M. Chauvin ${ }^{1}$ and D. J. Clark ${ }^{1,2}$ \\ Received ; accepted
}

\begin{abstract}
Operational since 2002 on-board the INTEGRAL observatory, the SPI spectrometer can be used to perform polarization measurements in the hard Xray/soft $\gamma$-ray domain $(\sim 130 \mathrm{keV}-8 \mathrm{MeV})$. However, this phenomenon is complex to measure at high energy and requires high fluxes. Cyg X-1 appears as the best candidate amongst the X-ray binaries since it is one of the brightest persistent sources in this energy domain. Furthermore, a polarized component has recently been reported above $400 \mathrm{keV}$ from IBIS data. We have therefore dedicated our efforts to develop the required tools to study the polarization in the INTEGRAL SPI data and have first applied them to $2.6 \mathrm{Ms}$ of Cyg X-1 observations, covering 6.5 years of the INTEGRAL mission.

We have found that the high energy emission of Cyg X-1 is indeed polarized, with a mean polarization fraction of $76 \% \pm 15 \%$ at a position angle estimated to $42^{\circ} \pm 3^{\circ}$, for energies above $230 \mathrm{keV}$. The polarization fraction clearly increases with energy. In the 130-230 keV band, the polarization fraction is lower than 20 $\%$, but exceeds $75 \%$ between 370 and $850 \mathrm{keV}$, with the (total) emission vanishing above this energy. This result strongly suggests that the emission originates from the jet structure known to emit in the radio domain. The same synchrotron process could be responsible for the emission from radio to $\mathrm{MeV}$, implying the presence of high energy electrons. This illustrates why the polarization of the high energy emission in compact objects is an increasingly important observational objective.
\end{abstract}

\footnotetext{
${ }^{1}$ Université de Toulouse; UPS-OMP; IRAP; Toulouse, France

CNRS; IRAP; 9 Av. colonel Roche, BP 44346, F-31028 Toulouse cedex 4, France

${ }^{2}$ Createc Ltd; Unit 8, Derwent Mill Commercial Park, Cockermouth, Cumbria, CA13 0HT, United Kingdom
} 
Subject headings: Gamma-rays:Binaries — Gamma-rays:individuals: Cyg X-1— Methods: observational-Polarization-Radiation mechanisms: general

\section{INTRODUCTION}

The high energy emission of X-ray binaries remains a debated topic. Between a few and 100-200 keV, a thermal inverse Comptonization of disk photons in a hot corona, accompanied by a disk reflection component, provides a good description of the data, but we are far from a complete understanding. In particular, some bright sources exhibit an excess of emission above 200-300 keV. This feature has been confirmed by several instruments operating above $300 \mathrm{keV}$, first in Cyg X-1 (see for example Nolan et al. 1981 or Liang \& Nolan, 1984) but also in a few other bright hard X-ray sources (i.e. GX 339-4 or the X-ray transient GRO J0422+32, see Bouchet et al., 1993; Johnson et al., 1993; Roques et al., 1994). The COMPTEL/CGRO instrument has shown that, in Cyg X-1, this component extends up to a few $\mathrm{MeV}$, with a power law slope around 2.6 or 3, depending on the source state (McConnell et al., 2002). This is often interpreted as a Comptonization process but with a non-thermal electron population, possibly linked to the thermal population. Alternative models based on jet emission also exist (see for instance Markoff et al. 2001). Indeed, it is clear now that many X-ray binary systems harbor (relativistic) variable radio jets and that radio and X-ray emissions are linked (Gallo et al. 2003).

Polarization measurements are a powerful tool to get specific information on the emission mechanisms and related parameters, as an effective supplement to spectral and timing studies. While common in radio or optical bands, they are less accessible in the high energy domain. However, two instruments aboard the INTEGRAL observatory allow such measurements. The IBIS imager obtains the polarization information from the Compton scattering occuring between its two separate detector planes (Forot et al. 2007). In comparison, the SPI spectrometer obtains the polarization from the scattering of photons within its large volume detector plane composed of 19 independent germanium crystals.

First measurements have been performed by both instruments independently on the Crab pulsar (Dean et al, 2008; Forot et al, 2008) and a strong gamma-ray burst, GRB041219A

\footnotetext{
${ }^{1}$ Based on observations with INTEGRAL, an ESA project with instruments and science data centre funded by ESA member states (especially the PI countries: Denmark, France, Germany, Italy, Spain, and Switzerland), Czech Republic and Poland with participation of Russia and USA.
} 
(McGlynn et al. 2007; Götz et al. 2009), demonstrating that polarization information can and must be sought in the high energy domain.

Cyg X-1 is, with the Crab, one of the main sources in the hard X-ray/soft $\gamma$-rays sky. It has been observed in many occasions by various instruments in this domain and presents complex spectral variability around two main spectral states (Jourdain et al. 2012 and references therein). It is therefore an obvious candidate for high energy polarization measurements. Laurent et al. (2011) recently published the detection of polarized emission, leading to the most popular scenarios regarding the high energy emission of black hole Xray binaries to be significantly revisited. These polarization studies are expected to provide extra information to decipher the mechanisms of the central engine.

In this paper, we use SPI observations to investigate the polarized emission in the high energy spectrum of Cyg X-1. Section 1 describes the instrument, analysis principles and data set. We then present the results obtained and conclude by discussing the first constrains we can learn from them.

\section{INSTRUMENT, DATA ANALYSIS AND OBSERVATIONS}

The SPI spectrometer aboard the INTEGRAL observatory consists of a coded mask associated with a detector plane of 19 germanium crystals, imaging the sky between 20 $\mathrm{keV}$ and $8 \mathrm{MeV}$. The large field of view $\left(30^{\circ}\right.$ in diameter), coupled with a limited number

of pixels (19), results in the use of a dithering strategy for observations to increase the number of independant measurements taken of a region of sky. An observation becomes a series of exposures (or science windows, SCW) of 30-40 minutes duration, taken in a predetermined pattern of pointings, which changes how the mask shadow is projected on to the detector plane. The pointing direction between SCWs usually varies by $\sim 2.3^{\circ}$. Two or three observations (dedicated to different targets) are successively performed during one INTEGRAL revolution (or orbit). Each revolution lasts $\sim 3$ days, resulting in $\sim 2.5$ days of uninterrupted operating mode, when excluding the crossing of the radiation belts. A description of the SPI telescope as well as its global performance can be found in Vedrenne et al. (2003) and Roques et al. (2003).

To use the polarimetry capability of SPI requires going deeper into the detection process and corresponding data analysis than is usually required. When a photon interacts with the camera, it loses its energy via Compton scattering and/or photoelectric absorption. When the incident photon deposits energy within more than one pixel this is classified as a "multiple event", as opposed to a "single event" where the energy is deposited within a single pixel. 
It is the angular distribution of the scatter directions involved in the multiple events that allows the determination of the polarization information. Indeed, the Klein-Nishina cross section for a linearly polarised photon presents itself as a function of the polarisation angle (more precisely of $\cos ^{2} \eta$ ), with its maximum at $\eta=90^{\circ}$. However, due to the competition between photoelectric and Compton processes, the multiple events play a significant role only for photons above $\sim 130 \mathrm{keV}$, where they represent $\sim 20 \%$ of the detected photons for the SPI geometry.

Events are tagged as "multiples" when they occur in (at least) 2 detectors, within a time-window of 350 ns. For these events, we are able to reconstruct the initial energy by adding the measured deposits, while the scattering direction is given by the identification of the involved detectors. The imaging information is contained here in 42 pairs of adjacent detectors (called pseudo-detectors), which play the same role as the 19 individual crystal for the single events. For any observation, the events are extracted in a similar way than for the single events (Jourdain et al. 2009), but using the imaging transfer matrix for the multiple events. The background shape is determined during empty field observations, and its normalization factor is allowed to vary during fitting on a six hours timescale. The source flux is considered constant on the revolution (ie $\sim 1-3$ days) timescale. The source and background fluxes are obtained for each energy band through a model fitting procedure convolving the sky model with the transfer matrix.

By carrying out this procedure in a number of channels, we can build the spectrum of the source, based on the multiple events. Similarly to the standard (single events) data procedure, the count spectrum is related to the incident photon spectrum through the appropriate (ground calibrated, see Attié et al., 2003) redistribution matrix, used in the spectral deconvolution step. This pipeline has been first validated using Crab Nebula observations. The total spectrum obtained for the Crab with $\sim 600 \mathrm{ks}$ of data has been found to be perfectly compatible with the result deduced from the standard analysis chain between $20 \mathrm{keV}$ and $3 \mathrm{MeV}$, making us confident in our analysis method and response matrices.

The response matrices used in the spectral analysis have been built for an unpolarized emission. However, the instrument response varies with the polarization of the incident flux, since the polarization introduces an anisotropy in the scattered photons. We have therefore replaced the unpolarized (ie averaged) matrix response with a polarized response in any study aiming to recover the polarization information, such as this. A dedicated paper (Chauvin et al., submitted) explains in detail the polarimetry technique used in the present analysis, which we summarize here.

An incident polarized photon preferentially scatters perpendicular to its polarization vector. This scattering forms a probability distribution that requires the observation of 
many photons to determine the original polarization vector.

For the polarization analysis, the imaging transfer function is computed at each incident direction and for each polarization angle, thanks to a detailed SPI mass model based on the Geant4 software.

For a given pointing, simulations are carried out for 18 polarization angles $\left(0-170^{\circ}\right.$ in $10^{\circ}$ steps, $100 \%$ polarized) and one unpolarized. To introduce a partial polarization fraction, a set of data is built by mixing the polarized and unpolarized simulated distributions:

$$
G 4\left(f_{p}, \Pi\right)=\frac{f_{p} \times G 4(\Pi)}{100}+\frac{\left(100-f_{p}\right) \times G 4(\varnothing)}{100}
$$

where $G 4\left(f_{p}, \Pi\right)$ represents the (Geant4) simulated data for a $f_{p} \%$ polarized flux at an angle $\Pi, G 4(\Pi)$ is the $100 \%$ polarized simulated data at angle $\Pi$ and $G 4(\varnothing)$ the unpolarized simulated data.

For each exposure, we compare the observed data and the simulated patterns, allowing two free parameters: the source flux and the background normalizations. They are determined through a linear least squares resolution which minimizes the expression:

$$
\begin{gathered}
\chi^{2}\left(f_{p}, i\right)=\sum\left(M_{f_{p}, i}(E)-D(E)\right)^{2} / D(E)^{2} \\
M_{f_{p}, i}=x \times G 4\left(f_{p}, \Pi\right)+y \times B
\end{gathered}
$$

where $\mathrm{x}$ is the source normalization, G4, the simulated count distribution, $\mathrm{y}$ is the background normalization and B,the background spatial distribution, over the energy band considered (E). The source model $G 4_{s d}\left(f_{p}, \Pi\right)$ describes the number of counts as a function of source polarization fraction, $f_{p}$, and angle, $\Pi$.

In a global data analysis, all the selected pointings are processed simultaneously, for each polarization angle and $f_{p}$ value, to give a final $18 \times 101 \chi^{2}$ array. Among them, the lowest value identifies the most probable (polarization angle, polarization fraction) parameter values.

The errors on the 2 free parameters have also been estimated through Monte-Carlo simulations with 1000 iterations for each configuration. The obtained distributions are centered around the simulated values (angle or fraction) and their widths correspond to values given by the $\chi^{2}$ statistics criterion $\Delta \chi^{2}=\chi^{2}+1$, which has been used for the remainder of the analysis. More details on the method, Monte-carlo simulations, analysis procedure and validation tests are given in a forthcoming paper (Chauvin et al., submitted). Note that our simulation code is based on an improved version of the code used in previous polarization studies (McGlynn et al. 2007, Dean et al. 2008), while the data analysis procedure has been developed independently and includes specific parameters, for the background handling for 
exemple. We have started our Cyg X-1 analysis with the same dataset presented for the spectral analysis of Jourdain et al. (2012), which covers 2003 June - 2009 December period. In spite of an increase of the flux by more than a factor two or three and a clear spectral variability, the source was mainly in its hard state, with some incursions into the intermediate ones. Since the multiple events concern only $\sim 20 \%$ of the total flux, the polarization analysis suffers from much weaker statistics. Moreover, for a given incident direction, the evolution of the projected pattern with the polarization angle is small. This implies that the sky model must be as simple as possible (only one source), and leads us to exclude some periods where the transient source EXO 2023+375, close to Cyg X-1, flares. Revolutions presenting a too high normalization background have also been removed. Finally, we present here the analysis of a data set limited to $2.6 \mathrm{Ms}$ of effective time, corresponding to 28 INTEGRAL revolutions, distributed over 6.5 years (see Tab. 1).

\section{RESULTS}

We analyzed the total dataset presented in Table 1 , and we initially considered the energy band from 230 to $850 \mathrm{keV}$. The photons above $850 \mathrm{keV}$ have not been included since the flux in this energy range appears very weak in the spectral analysis of the source (Jourdain et al. 2012) and decreases the significance of the result. As described above, for a given energy band, we compare the spatial distribution of the photons recorded on the detector plane and in the simulation, letting the flux of the source and background to vary on the revolution and 6 hours timescales, respectively. The results are presented in Figure 1. The displayed surface corresponds to the $\chi^{2}$ values obtained from eq. (1), in the (position angle, polarization fraction) space. Additionally, we also plot (right panel) the best polarization fraction (given by the lowest $\chi^{2}$ value) for each of the tested polarization angles. The $\chi^{2}$ surface appears smoothly structured with a trough, identifying the best fit between the observations and the simulated data. In the top projection, a contour plot illustrates more clearly the $\chi^{2}$ behavior around its minimum. The three grey level areas are associated to the 1 (2 and 3$) \sigma$ levels, as deduced from the $\chi 2=\chi 2+2.3(+6.2,+11.8$ resp.) formula.

We see that the observations are better described by an incident emission with a polarization fraction of $76 \% \pm 15 \%$ at a position angle of $42^{\circ} \pm 3^{\circ}$.

We have also performed the same analysis in three energy bands: $130 \mathrm{keV}-230 \mathrm{keV}, 230$ $\mathrm{keV}-370 \mathrm{keV}$ and $370 \mathrm{keV}-850 \mathrm{keV}$. The results are presented on Figures 2, 3 and 4 . In the first energy range, the $\chi^{2}$ surface is quite flat, implying that no significant polarization is detected. The situation appears quite different when looking at higher energy. In the 230-370 keV and $370-850 \mathrm{keV}$ bands, the $\chi^{2}$ surfaces present a clear minimum around a 
polarization angle of $40^{\circ}-50^{\circ}$, with an increasing polarization fraction. The contour plots (top panels) give the best fit values together with their errors. The position angles are rather well determined: $47^{\circ} \pm 4^{\circ}$ and $39^{\circ} \pm 3^{\circ}$, in the medium and high energy band respectively. The polarization fraction is less easy to constrain. Nonetheless, we clearly see that it increases with energy, being undetectable at low energy, ( $3 \sigma$ upper limit of $20 \%$, assuming the position angle determined above), it reaches $41 \% \pm 9 \%$ in the medium band, and is constrained to be greater than $75 \%$ at a $2 \sigma$ level at higher energy.

These results show that the high energy part of the Cyg X-1 emission is polarized, with a polarization fraction increasing with energy. More precisely, we find that the polarization is present down to $\sim 230 \mathrm{keV}$, whilst it becomes hard to detect below that energy. This implies that the hard X-ray/soft $\gamma$-ray emission presents different properties below and above $\sim 230 \mathrm{keV}$. This fits with the spectral behaviour seen in Cyg X-1, where a component in excess of the thermal Comptonization appears above $\sim 250 \mathrm{keV}$. It is straightforward to link these two results and associate the second component to the polarizing mechanism. Given the high polarization fraction observed, a likely scenario relies on the synchrotron radiation in a very ordered magnetic field as the mechanism responsible of the high energy tail.

This information can be introduced in the description of the observed spectral shape. We combine the spectrum extracted from the single events (standard analysis), which provides the low energy emission (down to $22 \mathrm{keV}$ ), and the spectrum from the multiple events. A two component model is fit to the spectra using Xspec11. The first component, a thermal Comptonization model, Comptt (plus reflection) is used to describe the low energy part. The high energy component is represented by a cutoff power law, tentatively identified with the synchrotron process. Since the limited significance of the high energy data prevents us actually constraining the corresponding parameters, we have fixed the cutoff power law with a photon index to 1.6, and the cutoff value to $\sim 700 \mathrm{keV}$. These hypotheses result in a good reproduction of the global spectrum. Fig 5 displays the resulting breakdown of the observed spectral emission, in the proposed framework. Both components intersect around $240 \mathrm{keV}$, in agreement with a scheme where a polarized radiation emerges above this energy while the non polarized Comptonized flux dominates at lower energy.

\section{DISCUSSION}

The recent detection by INTEGRAL IBIS (Laurent et al. 2011) of a strongly polarized emission above $400 \mathrm{keV}$ in the X-ray binary Cyg X-1 emission can be seen as the beginning of a new era for the X-ray binaries high energy emission topic. Our results, based on SPI 
data, confirm the polarization of the high energy emission, and allow a more precise picture.

We find that the emission presents a strong polarization above $230 \mathrm{keV}$ with a position angle of $42^{\circ} \pm 3^{\circ}$ East from the North. We have to note that this value is clearly incompatible with the IBIS/Compton mode result reported by Laurent et al (2011) of $140^{\circ} \pm 10^{\circ}$. However, due to an error in the angle convention, the correct value for the IBIS /Compton mode is $180-140^{\circ}=40^{\circ}$, (P. Laurent, private communication), in perfect agreement with that presented here.

Both analyzes report an increase of the polarization fraction. In the SPI data, while only upper limits are accessible below $230 \mathrm{keV}\left(f_{p}<20 \%\right.$ at a $3 \sigma$ level $)$, the emission at higher energy presents a strong polarization fraction : $40 \pm 10 \%$ and then $>75 \%$ above $400 \mathrm{keV}$. High degrees of polarization are usually expected from a synchrotron process. Generally, polarization requires a very ordered configuration with a single axis geometry or collimated structure.

From radio observations, Cyg X-1 is known to present some persistent jet emission, when in the hard state, (Stirling et al. 2001), as well as transient emission (Fender et al. 2006). The reported jet orientations are around $-20^{\circ}$ or $-25^{\circ}, \sim 65^{\circ}$ away (onto the sky), from the polarization angle deduced for the high energy photons. These values are of prime importance to investigate the acceleration process and better comprehend the origin of the photons.

Combined with the spectral analysis, the polarization measurements provide crucial extra information in the global picture of the hard X-ray emission of Cyg X-1. It has been known for a long time that the spectral shape observed at high energy for Cyg X-1 requires the presence of two components. Similarly, the evolution of the polarization fraction with energy is expected in a scenario where two mechanisms coexist in the hard X-ray/soft $\gamma$-ray domain and intersect around 2-300 keV. We can establish a strong link between the second component and the polarizing mechanism, and suggest that in this source, the thermal Comptonization, unpolarized, component largely dominates the global emission up to 200 or $300 \mathrm{keV}$ before giving way to a second, polarized, process. The statistics limit the precision of our analysis but it is interesting to note that, in principle, precise polarization measurements would allow both mechanisms to be disentangled, if we assume that one is totally polarized (or have a high enough energy independent polarization fraction). Conversely, if the polarization fraction behavior is intrinsic to the emission mechanism, its study will help constrain it.

The synchrotron process represents the most probable way to produce such strongly polarized emission. A scenario based on optically thin synchrotron emission from a partially self absorbed jet has already been studied by Zdziarski et al. (2012). They fail to easily reconcile their model with the data, because they impose a unique broken power law to 
explain the source emission from radio to high energy. However, the SPI data cover a 6.5 year period, while the radio or IR observation are very short. We therefore consider SPI data alone to estimate the parameters in the synchrotron framework. Even if it is difficult to put firm constrains, some rough features can be deduced. In particular, the emitted photon energy distribution is directly connected to the electron population parameters (see Ginzburg and Sirovatski, 1965). Similarly to Zdziarski et al. (2012), we find that a rather hard (photon) slope is needed, since its contribution should decrease toward X-ray band. Finally, a good description of the second (synchrotron) component can be obtained with a photon index of 1.6, corresponding to the reasonable value of 2.2 for an electron power law population. More information is potentially hidden in the observed cutoff energy (although not very constrained). We have fixed a cutoff value of $700 \mathrm{keV}$, slightly less than the folding energy obtained by Zdziarski et al. (2012), which overestimated the MeV emission. While the photon-photon absorption cannot be excluded, such a feature is more probable due to the similar rollover in the emitting electron distribution. However, a more precise determination depends on the magnetic field strength, and requires some additional inputs or/and deeper modeling work.

A point worth mentioning is that the detection of such a signal implies its stability over long periods, and consequently, the same stability of the emitting medium. Whatever the mechanisms at work, they must keep (or come back to) the same characteristics. This should include the process behind the relativistic electron acceleration.

\section{SUMMARY AND CONCLUSIONS}

We have presented our analysis into the polarization phenomenon, through its role in the hard X-ray/soft $\gamma$-ray of X-rays binaries. The analysis of the multiple events in the observations of Cyg X-1 from the SPI instrument refines (and corrects) the first result reported by Laurent et al. (2011) from the IBIS/Compton mode. It shows that the source emission is strongly polarized above $\sim 200 \mathrm{keV}$, with the fraction of polarization increasing with energy up to $850 \mathrm{keV}$. This behavior can be understood in terms of two emission mechanisms, one polarized and the other not, which contribute simultaneously to the hard X-rays. The non polarized process would dominate at low energy, cutting off above 150 $\mathrm{keV}$ and is well explained by thermal Comptonization. The polarized emission presents a harder spectral shape providing higher energy photons up to $\sim 1 \mathrm{MeV}$. This consolidates the picture emerging from all high energy instruments that could not be reconciled with a single Comptonization law.

Building on this idea, we have performed a spectral study from $20 \mathrm{keV}$ to $1 \mathrm{MeV}$, gath- 
ering the simultaneous standard SPI data (single events) and the multiple events analyzed in this paper. At low energies, the Comptonization of the disk photons by hot electrons explains both the spectral shape and the low/null polarization level of the emission. The excess of emission observed at high energies above this thermal Comptonization law can be associated with the polarization phenomenon and may be described by a cutoff power law of photon index 1.6 with a cutoff energy (poorly constrained) around $700 \mathrm{keV}$.

Since the polarization requires a very organized medium to exist, a highly polarized emission is generally associated to a jet. Thus it is likely that the high energy tail observed in the Cyg X-1 spectrum is not associated to the disk but to an electron population accelerated somewhere in the jet, now well established to exist in this source from radio observations. The position angle deduced from the analysis $\left(42^{\circ} \pm 3^{\circ}\right)$ is oriented $\sim 60^{\circ}$ away from the radio jet direction. An important point to note is that the polarization detection, particularly at such a high degree, implies that the phenomenon is persistent on a timescale of weeks to months.

To conclude, the detection of a polarized high energy emission in Cyg X-1 significantly impacts our view of X-ray binaries. A new scenario is now preferred where the jet structure that has been shown to exist in this class of source, can now be shown to perform a major role in the observed emission in the hard X-ray/soft $\gamma$-ray domain, a feature in many jet based models.

This new window opened into our understanding of X-ray binaries also strengthens the link between stellar mass black hole binaries and AGN characteristics. This will enable us to learn a lot from studies of both Galactic and extragalactic populations. Polarization measurements of many more sources will be required to build upon this picture. Measurements are strongly limited by the capabilities of the current generation of instruments which were not designed with polarimetry in mind. So to significantly progress this field, future missions should be planned to include a polarization ability that can help us understand the high energy environment in astronomical objects and break degeneracies between emission mechanisms in theoretical models.

\section{Acknowledgments}

The INTEGRAL SPI project has been completed under the responsibility and leadership of CNES. We are grateful to ASI, CEA, CNES, DLR, ESA, INTA, NASA and OSTC for support. MC and DC gratefully acknowledge financial support provided by the CNES, and we thank the anonymous referee for interesting exchanges. 


\section{REFERENCES}

Attié D., Cordier B., Gros M., et al, 2003, A\&A, 411, L71

Bouchet, L., Jourdain, E., Mandrou, P. et al., 1993, ApJ, 407, 739

Dean, A. J., Clark, D. J., Stephen, J. B., et al., 2008, Science, 321, 1183

Fender, R. P.; Stirling, A. M.; Spencer, R. E. et al., 2006, MNRAS, 369, 603

Forot, M., Laurent, P., Lebrun, F. and Limousin, O., 2007, ApJ, 668, 1259

Forot, M., Laurent, P., Grenier, I., Gouiffes, C. and Lebrun, F. , 2008, ApJ, 688, 29

Gallo, E., Fender, R. P. and Pooley, G. G., 2003, MNRAS, 344, 60

Ginzburg, V. L. and Syrovatskii, S. I., 1965, Ann. Rev. Astron. Astrophys., 3, 297

Götz, D., Laurent, P., Lebrun, F., Daigne, F. and Bovnjak, Z. ,2009, ApJL, 695,208

Johnson, W. N., Kurfess, J. D., Purcell, W. R. et al., 1993, A \& AS, 97, 21

Jourdain, E. \& Roques, J. P., 2009, ApJ, 704, 17

Jourdain, E., Roques, J. P.\& Malzac, J., 2012, ApJ, 744, 64

Laurent, P., Rodriguez, J., Wilms, J et al., 2011, Science, 332, 438

Liang, E. P. and Nolan, P. L., 1984, SSRv, 38, 353

Markoff, S., Falcke, H. and Fender, R., 2001, A\&A, 372, L25

McGlynn, S., Clark, D. J., Dean, A. J., et al. 2007, A\&A, 466, 895

McConnell, M. L., Zdziarski, A. A., Bennett, K. et al., 2002, ApJ, 572, 984

Nolan, P. L., Gruber, D.E., Knight, F. K. et al., 1981, Nature, 293, 275

Roques J.P., Bouchet, L., Jourdain, E.., et al, 1994, ApJS, 92, 451

Roques J.P., Schanne S., Von Kienlin A., et al, 2003, A\&A, 411, L91

Stirling, A.M., Spencer, R. E., de la Force, C. J. et al., 2001, MNRAS, 327, 1273

Vedrenne, G., Roques, J.P., Schonfelder, V. et al, 2003, A\&A, 411, L63

Zdziarski, A. A., Lubinski, P. and Sikora M., 2012, MNRAS, 423, 663 
b)
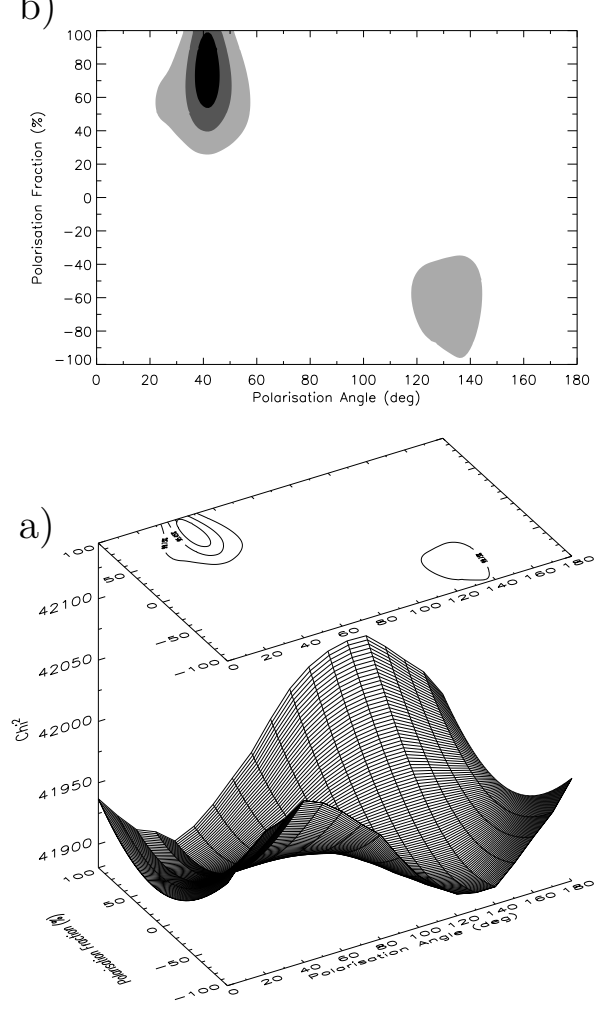

d)

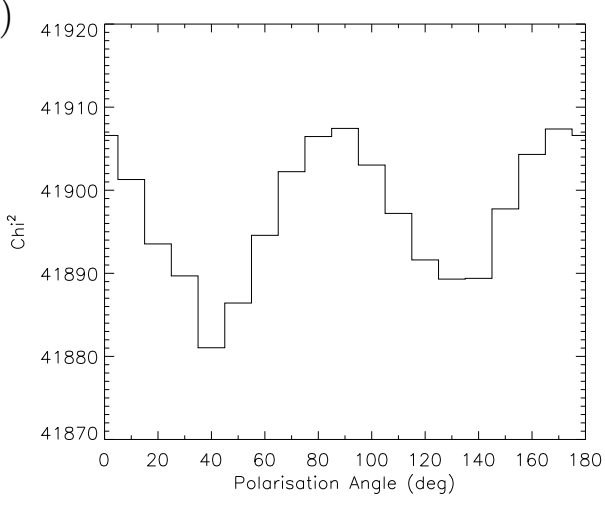

c)

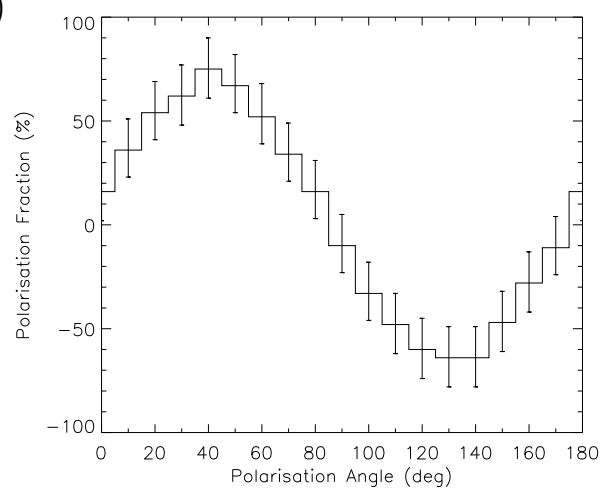

Fig. 1. - Left panel: a) $\chi^{2}$ map result versus position angle and polarization fraction in the $230-850 \mathrm{keV}$ band. b) The contour projections at $\Delta \chi 2=2.3,6.2,11.8$. Right panel: c) Polarization fraction giving the best fit for each position angle and d) the corresponding $\chi^{2}$ curve. 
Table 1. Log of the INTEGRAL SPI observations of Cyg X-1 used in this paper.

\begin{tabular}{lccc}
\hline \hline \multicolumn{1}{c}{$\begin{array}{c}\text { Sevol } \\
\text { number }\end{array}$} & Start & End & $\begin{array}{c}\text { useful } \\
\text { duration (ks) }\end{array}$ \\
& & & \\
\hline $79-80(5 \times 5)$ & $2003-06-07$ 00:59 & $2003-06-1203: 35$ & 293 \\
$210-214(\mathrm{~A})$ & $2004-07-0300: 01$ & $2004-07-1700: 25$ & 709 \\
$251-252(\mathrm{~A})$ & $2004-11-0314: 23$ & $2004-11-0716: 26$ & 176 \\
$259 \& 261(\mathrm{H})$ & $2004-11-2612: 28$ & $2004-12-0315: 43$ & 143 \\
$628-631(\mathrm{~A})$ & $2007-12-0419: 05$ & $2007-12-1521: 08$ & 388 \\
$673(\mathrm{~A})$ & $2008-04-1817: 41$ & $2008-04-1922: 09$ & 54 \\
$682-684(\mathrm{~A})$ & $2008-05-1408: 13$ & $2008-05-2219: 54$ & 304 \\
$803-806(\mathrm{~A})$ & $2009-05-1108: 27$ & $2009-05-2211: 32$ & 371 \\
$875\left(\mathrm{H}^{*}\right) \& 877(\mathrm{H})$ & $2009-12-1216: 18$ & $2009-12-1920: 57$ & 160 \\
\hline
\end{tabular}

Note. - In the first column, the letter after the revolution number indicates the dithering strategy used: $(5 \times 5)$ for the standard $5 \mathrm{X} 5$ pattern (see section 2$)$; (A) for a pointing strategy centered between Cyg X-1 and Cyg A region; $(\mathrm{H})$ for the hexagonal pattern and (GP) for a Galactic Plane scan. $\left(\mathrm{H}^{*}\right)$ During the rev 875 , the pointing strategy follows a pattern proposed by Wilms et al in their AO-7 proposal. All this information is available on the dedicated ESA site web http://integral.esa.int/isocweb. 
b)
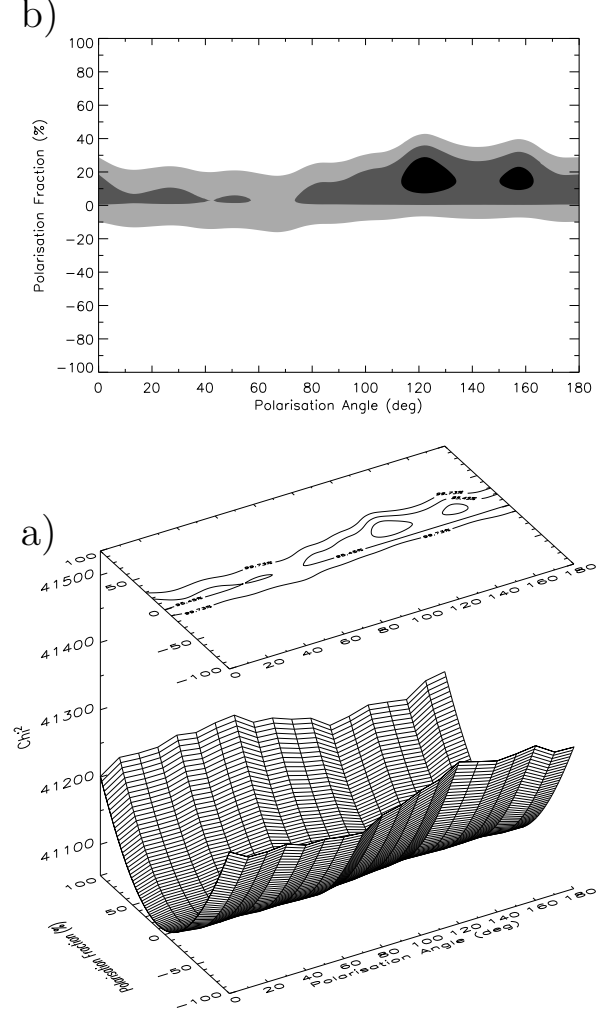

d)

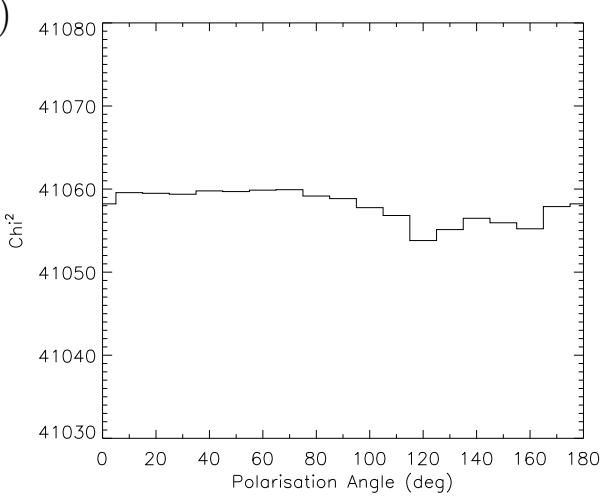

c)

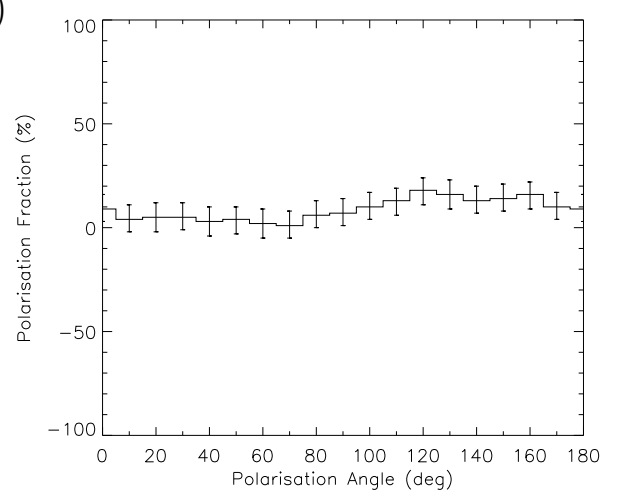

Fig. 2.- The same as Figure 1 for the 130-230 keV band. 
b)
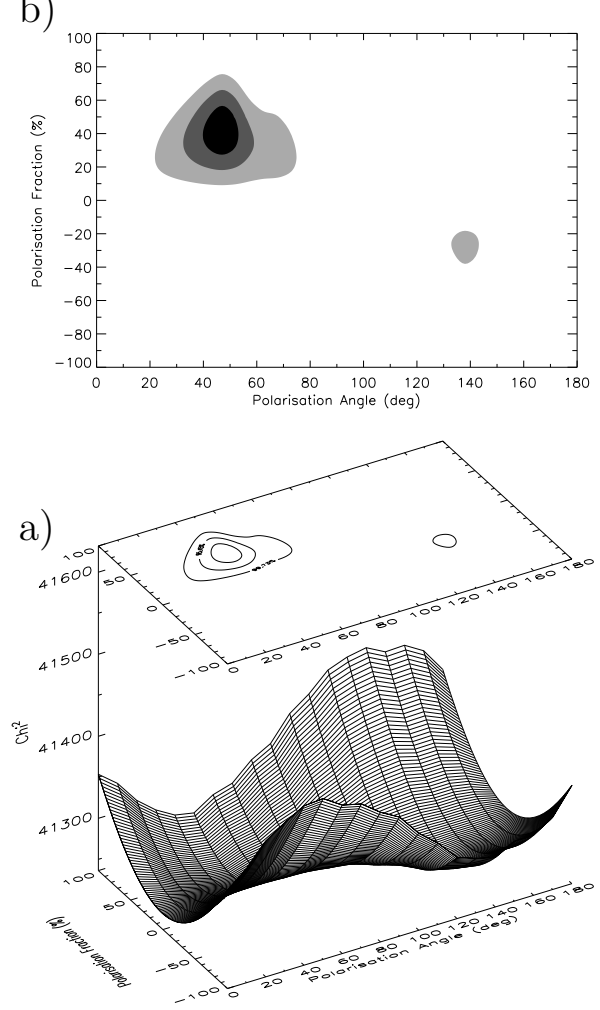

d)

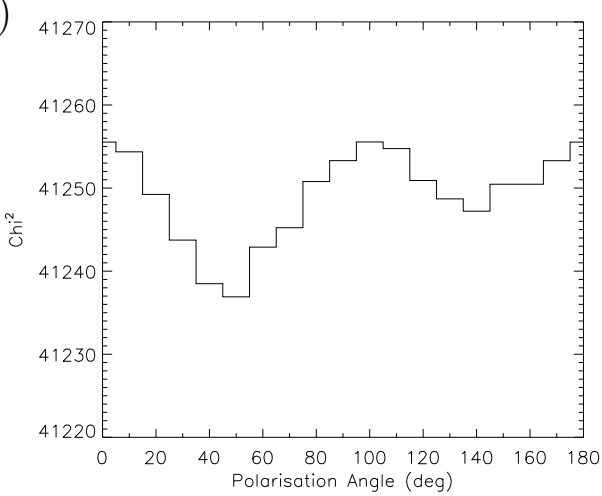

c)

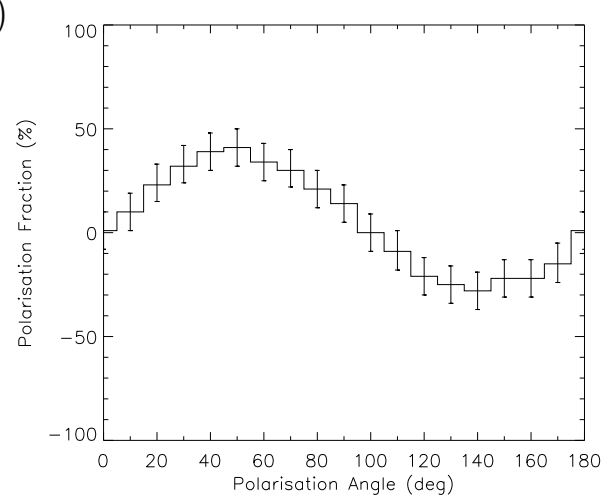

Fig. 3. - The same as Figure 1 for the 230-370 keV band. 
b)
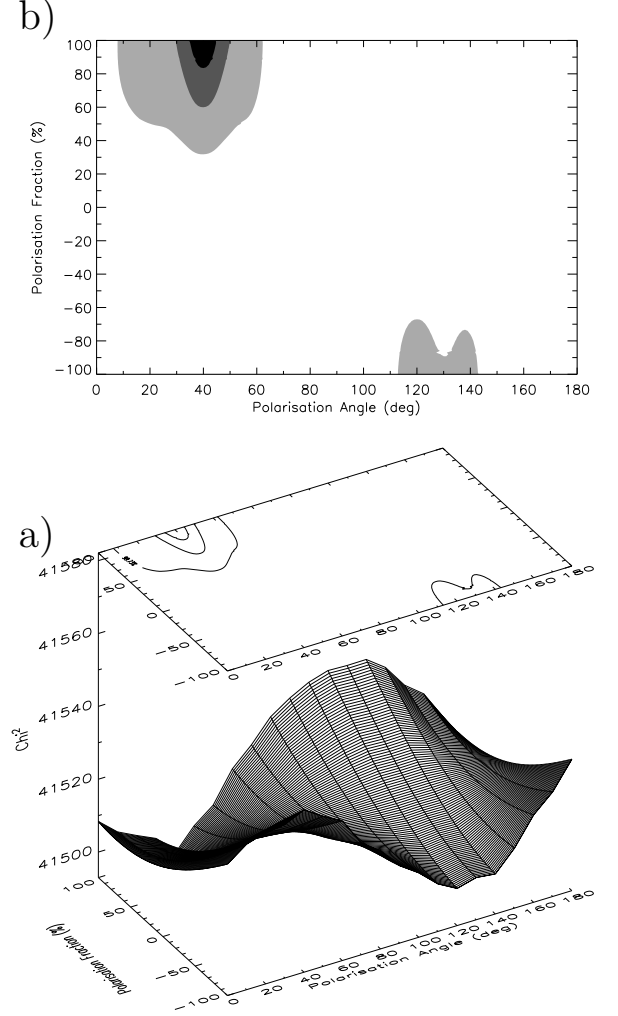

d)

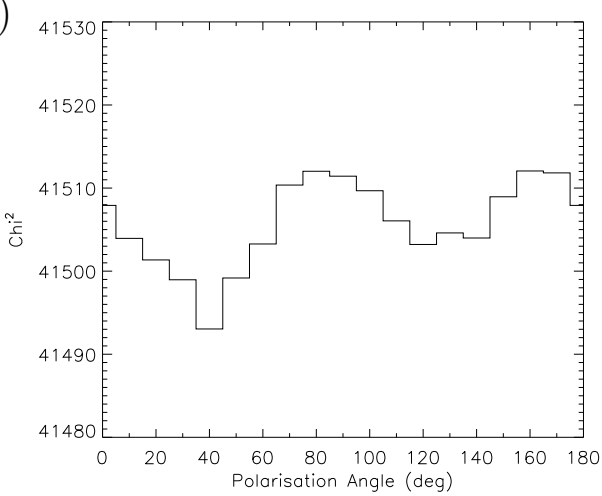

c)

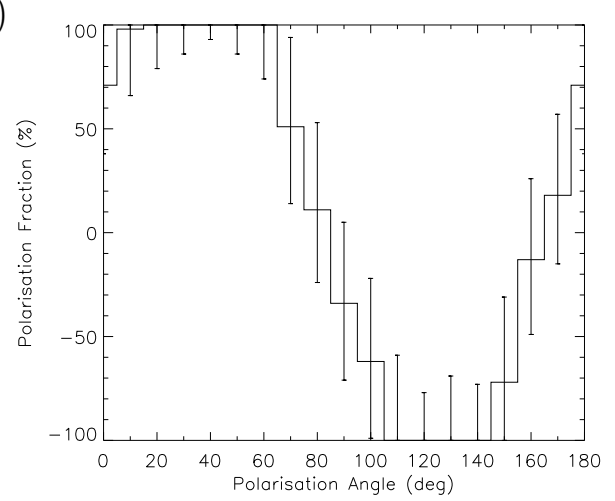

Fig. 4. - The same as Figure 1 for the $370-850 \mathrm{keV}$ band. 

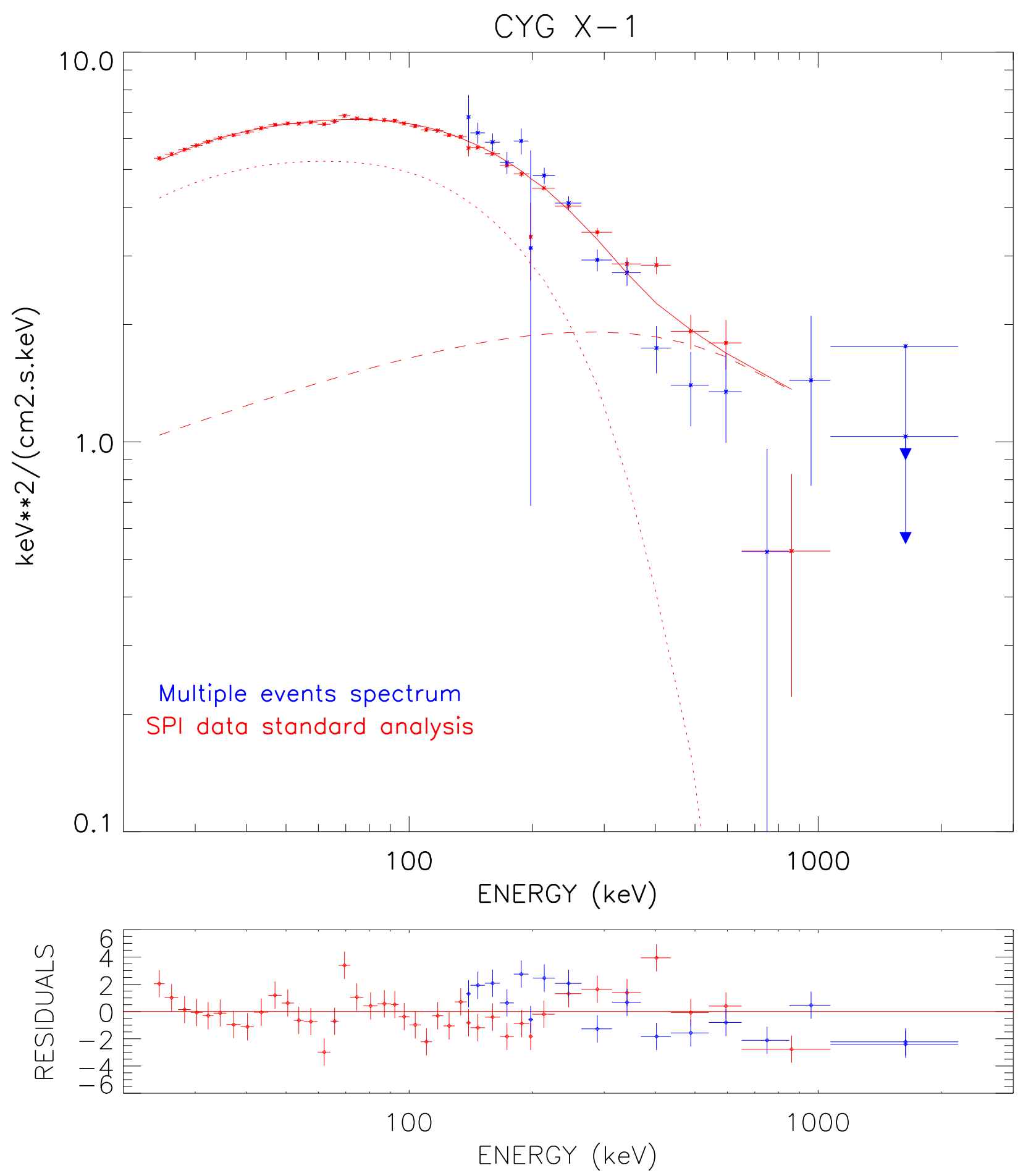

Fig. 5.- The stacked spectra of SPI obtained from standard analysis (single events; 22 keV-2 MeV) and from multiple events (130 keV-8 MeV). The solid lines represent the best fit model composed of a thermal Comptonization (reflec*CompTT, dotted curve) plus a fixed cutoff power-law model (photon index $=1.6, E_{\text {cut }}=700 \mathrm{keV}$; dashed line). $0.5 \%$ of systematic have been added to the data. 